\title{
Fast Inverse Laplace Transform for the Unsteady Thermal Response of Geothermal Heat Exchangers
}

\author{
Miguel Hermanns ${ }^{1}$ \\ ${ }^{1}$ Departamento de Mecánica de Fluidos y Propulsión Aeroespacial, Escuela Técnica Superior de Ingeniería \\ Aeronáutica y del Espacio, Universidad Politécnica de Madrid, Plaza Cardenal Cisneros 3, E-28040 Madrid, Spain \\ miguel.hermanns@upm.es
}

\begin{abstract}
The successful harnessing of geothermal energy for the efficient heating and cooling of buildings relies upon the correct sizing of geothermal heat exchangers. This is done during the building's design phase using extensive numerical simulations to forecast the unsteady thermal response of the geothermal heat exchanger and its surrounding ground for the next 25,50 , or even 100 years of operation. In the present work, an efficient numerical Laplace inversion formula is proposed, which applied to the obtained solution in the complex Laplace plane, allows the fast and accurate prediction of the sought unsteady thermal response.
\end{abstract}

\section{INTRODUCTION}

For the heating and cooling of buildings, the harnessing of geothermal energy represents one of the most energy efficient solutions available nowadays. An HVAC (heating, ventilation, and air conditioning) system based on that renewable energy source consists in a water-to-water heat pump connected to a geothermal heat exchanger composed of multiple vertical geothermal boreholes. Each of these boreholes is typically equipped with a U-shaped pipe through which a heat carrying liquid flows and exchanges heat with the surrounding ground. Such an HVAC system can only be considered truly renewable, however, if the geothermal heat exchanger is correctly designed and sized. Otherwise, an excessive drift in the operating temperatures of the heat carrying liquid builds up over time, negatively affecting the energy efficiency of the heat pump, and consequently of the whole HVAC system.

Advanced theoretical/numerical models are regularly used to study the unsteady thermal response of geothermal heat exchangers. One such model is the Superposition Borehole Model (SBM) that exploits the large disparity in time and length scales present in the equations to strongly simplify the three-dimensional unsteady heat conduction problem in the ground $[1,2]$. By numerically solving multiple axisymmetric unsteady heat conduction problems, with each one centered around each borehole, and then superimposing them, the SBM obtains the sought unsteady thermal response. In the original formulation of the model, those axisymmetric problems are discretized using finite differences for the spatial derivatives and an explicit Euler scheme for the time derivative.

In recent years, the SBM has been reformulated in terms of point sources of heat, allowing the problem and its solution to be expressed in terms of integral operators instead of differential equations $[3,4,5,6]$. Additionally, the Laplace transform is used to convert the time-marching problem into an algebraic problem, whose solution is much simpler to obtain [3,6]. Once the results in the complex Laplace plane are known, the sought time evolution of the geothermal heat exchanger is recovered through the inverse Laplace transform. The benefit of all this is a significantly reduced computational cost, with only 790 seconds of wall-clock time required on an Intel Core i5-2500K processor to obtain the unsteady thermal response of a typical geothermal heat exchanger composed of 100 boreholes [3].

Further reductions in computational time can be achieved by improving the employed numerical Laplace inversion formula. In [3], a method based on the Fast Fourier Transform is used, which needs to be invoked for each time instant to compute. In the present work, a different numerical Laplace inversion formula is developed that allows the unsteady thermal response of a typical geothermal heat exchanger composed of 100 boreholes to be obtained in just 206 seconds using a single core of an Intel Core $17-7700 \mathrm{~K}$ processor. Even after factoring out the performance difference between the two microprocessors, the proposed numerical Laplace inversion formula still leads to a three-fold speedup, with its computational cost not depending on the total number of time instants to compute. 


\section{THE SUPERPOSITION BOREHOLE MODEL IN THE COMPLEX LAPLACE PLANE}

A geothermal heat exchanger is composed of $N_{b}$ vertical geothermal boreholes. Each borehole $j$ is characterized by its nondimensional depth $h_{j}$, its nondimensional buried depth $d_{j}$, its slenderness $\Lambda_{j}$, its network of nondimensional thermal resistances $\left(A_{j}, B_{j}\right.$, and $\left.S_{j}\right)$, and its nondimensional operating conditions $\Omega_{j}[4,5,7]$. The thermal state of each borehole $j$ is given by the nondimensional temperature difference $\Delta_{j}$ between the two legs of the U-shaped pipe, the nondimensional weighted mean fluid temperature $\Theta_{j}$, and the nondimensional heat injection rate per unit borehole length $\bar{q}_{j}$, all of which are functions of the nondimensional vertical coordinate $\xi$ and the nondimensional time $\tau$ [6].

To obtain the functions $\Delta_{j}(\xi, \tau), \Theta_{j}(\xi, \tau)$, and $\bar{q}_{j}(\xi, \tau)$, the unsteady heat conduction in the ground and the grout and the unsteady convective heat transport along the U-shaped pipes need to be modeled and solved. Using the simplifying assumptions and hypotheses of the Superposition Borehole Model, and the Laplace transform to address the resulting time-marching problem, the following set of equations is obtained for each borehole $j[4,5,6,7]$ :

$$
\frac{d \tilde{\Delta}_{j}}{d \xi}=-\tilde{q}_{j}, \quad \frac{d \tilde{\Theta}_{j}}{d \xi}=\frac{\Omega_{j} S_{j}}{h_{j}} \tilde{q}_{j}-\frac{\Omega_{j}^{2}}{h_{j}^{2} A_{j}} \tilde{\Delta}_{j}, \quad \tilde{\Theta}_{j}-\tilde{\Theta}_{b j}=\tilde{q}_{j} B_{j},
$$

where the superscript $\sim$ denotes the Laplace transform of the corresponding variable. The nondimensional mean azimuthal borehole wall temperature $\tilde{\Theta}_{b j}(\xi)$ of borehole $j$, that accounts for the thermal response of the ground and for the thermal influences exerted by the neighboring boreholes, is given by

$$
\tilde{\Theta}_{b j}=\tilde{\Theta}_{\infty}+\sum_{n=1}^{N_{b}} \frac{1}{4 \pi} \int_{d_{n}}^{h_{n}} \tilde{q}_{n}\left(\xi_{0}\right)\left[\frac{\mathrm{e}^{-\sqrt{s} \sqrt{\eta_{j n}^{2}+\left(\xi-\xi_{0}\right)^{2}}}}{\sqrt{\eta_{j n}^{2}+\left(\xi-\xi_{0}\right)^{2}}}-\frac{\mathrm{e}^{-\sqrt{s} \sqrt{\eta_{j n}^{2}+\left(\xi+\xi_{0}\right)^{2}}}}{\sqrt{\eta_{j n}^{2}+\left(\xi+\xi_{0}\right)^{2}}}\right] \mathrm{d} \xi_{0},
$$

where $\tilde{\Theta}_{\infty}(\xi)$ is the nondimensional unperturbed ground temperature. The nondimensional planar distance between boreholes $j$ and $n$ is $\eta_{j n}$, with $\eta_{j j}=h_{j} / \Lambda_{j}$, and $s$ is the complex number in the Laplace plane.

Each of the $N_{b}$ boreholes is subject to the following boundary conditions at its top, $\xi=d_{j}$, and its bottom $\xi=h_{j}$,

$$
\xi=d_{j}: \quad \tilde{\Delta}_{j}=\tilde{f}_{j}, \quad \xi=h_{j}: \quad \tilde{\Delta}_{j}=0,
$$

with $\tilde{f}_{j}$ being the nondimensional heat injection rate of borehole $j$. The values of $\tilde{f}_{j}$ result from imposing a set of global constraints, being the first one the energy conservation at geothermal heat exchanger level [5]:

$$
\tilde{f}=\sum_{j=1}^{N_{b}} \tilde{f}_{j},
$$

where $\tilde{f}$ is the nondimensional heat injection rate imposed by the HVAC system onto the geothermal heat exchanger. Further constraints emerge from the condition that the heat carrying liquid fed to the boreholes comes from a horizontal network of distribution pipes. In case all boreholes are connected in parallel to that network, all the borehole inlet temperatures must be equal to the a priori unknown nondimensional inlet temperature $\Theta_{\text {in }}(\tau)$. This leads to the following set of $N_{b}$ constraints to be satisfied by the solution in the complex Laplace plane [5]:

$$
\tilde{\Theta}_{j}\left(d_{j}\right)+\frac{\Omega_{j}}{2 h_{j}}\left(S_{j}+1\right) \tilde{\Delta}_{j}\left(d_{j}\right)=\tilde{\Theta}_{\mathrm{in}}, \quad j=1, \ldots, N_{b} .
$$

The solution to the integro-differential system of equations formed by (1), (2), (3), (4), and (5) has to be obtained numerically for each value of the complex number $s$. To correctly capture the strong variations close to the borehole endings of the nondimensional heat injection rates per unit borehole lengths $\tilde{q}_{j}$, a grid point distribution based on the extrema of the Chebyshev polynomials of the first kind is used, where $N+1$ is the total number of grid points:

$$
\xi_{i j}=d_{j}+\left(h_{j}-d_{j}\right) \frac{1-\cos \left(\pi \frac{i}{N}\right)}{2}, \quad i=0, \ldots, N .
$$

By approximating the derivatives using first order finite differences and the integrals using the trapezoidal rule, a large system of linear algebraic equations is constructed. Its solution is obtained using an iterative GMRES solver, whose convergence is strongly accelerated using a physics-based preconditioner analogous to the one employed by the author in [8], thereby completing the formulation and solution of the SBM in the complex Laplace plane. The sought time evolution of the geothermal heat exchanger is then obtained by applying the inverse Laplace transform. 


\section{INVERSE LAPLACE TRANSFORM}

Given the function $F(s)$, that represents any of the results of interest that arise from the solution procedure described in the previous section, its temporal counterpart $f(\tau)$ is obtained using the inverse Laplace transform. In the present work, this inverse transformation is expressed through the Bromwich integral defined as

$$
f(\tau)=\frac{1}{2 \pi i} \lim _{T \rightarrow \infty} \int_{\sigma-i T}^{\sigma+i T} F(s) \mathrm{e}^{s \tau} \mathrm{d} s,
$$

where $i=\sqrt{-1}$ and $\sigma$ places the integration contour to the right of all singularities of $F(s)$. In order to exploit this expression, the integral along the line $\operatorname{Re}(s)=\sigma$ needs to be discretized using any of the well known quadrature rules. The convergence of the so obtained numerical Laplace inversion formula can be vastly improved by deforming the integration path. The aim is to exploit the exponential factor of the integrand by choosing a new path along which it decays rapidly, so that a reduced number of discretization points is required by the quadrature rule. This idea is valid as long as the new integration path is also located to the right of all singularities of $F(s)$.

Several different integration paths $z(\theta)$ have been proposed in the literature, with $\theta$ being the argument of the complex number $s[9,10,11]$. All of them share the property that $\operatorname{Re}(z( \pm \pi))=-\infty$, which ensures the rapid decay of the aforementioned exponential factor. Application of $z(\theta)$ to (7) leads to an integral between $-\pi$ and $\pi$, which can then be discretized, as shown in Figure 1, using the midpoint rule and the $2 N$ uniformly-spaced discretization points $\theta_{k}=\pi(2 k-1) /(2 N)$, with $k$ ranging from $1-N$ till $N$. Since the function $F(s)$ satisfies that $F\left(s^{*}\right)=F(s)^{*}$, where the superscript $*$ represents the conjugate of a complex number, the final expression for the numerical Laplace inversion formula is

$$
\begin{aligned}
f(\tau) & =\frac{1}{2 \pi i} \int_{-\pi}^{\pi} F(z(\theta)) \mathrm{e}^{z(\theta) \tau} z^{\prime}(\theta) \mathrm{d} \theta \\
& =\frac{1}{N} \sum_{k=1}^{N} \operatorname{Im}\left[F\left(z\left(\theta_{k}\right)\right) \mathrm{e}^{z\left(\theta_{k}\right) \tau} z^{\prime}\left(\theta_{k}\right)\right] .
\end{aligned}
$$

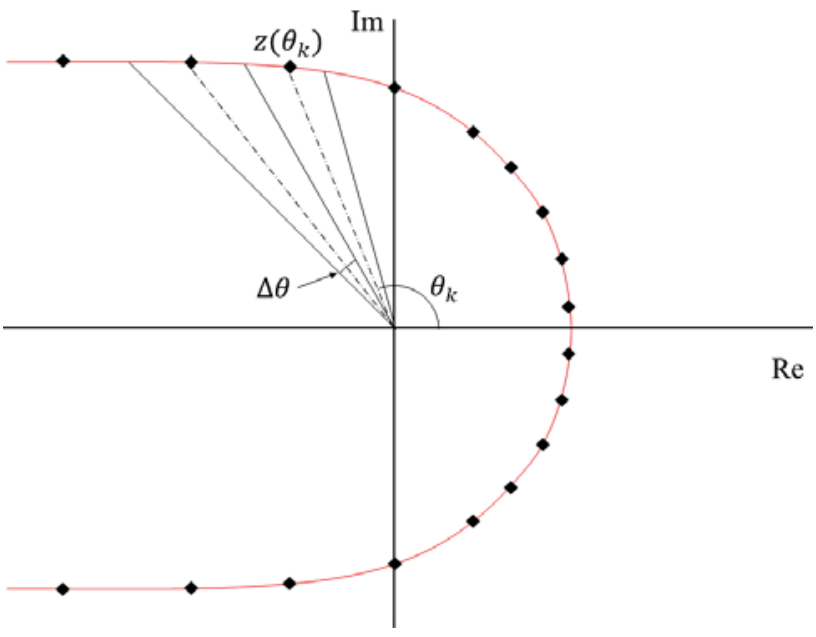

FIGURE 1. Sketch of the deformed integration path $z(\theta)$ with the employed uniformly-spaced discretization points $\theta_{k}$.

The number of evaluations $N$ required for a certain error level $\varepsilon$ depends on the chosen integration path. In the present work, the path proposed by Dingfelder and Weideman is used [11], for which $\varepsilon \approx \mathrm{e}^{-2.719 N}$. Hence, for single precision accuracy $\left(\varepsilon=10^{-8}\right)$, just $N=7$ evaluations would be required. Nevertheless, since the chosen integration path depends on the considered time instant $\tau$,

$$
z(\theta)=\frac{2 N}{\tau}\left(-0.6122+0.5017 \frac{\theta}{\tan (0.6407 \theta)}+0.2645 i \theta\right),
$$

and if $N_{\tau}$ time instants are to be computed, a total of $N_{\tau} N$ evaluations of the costly function $F(s)$ result, which can still be too computationally expensive.

In the present work a novel strategy is proposed to overcome the just highlighted problem. The idea is to use the same integration path for multiple time instants that are not too far apart, so that the same $N$ evaluations of the costly function $F(s)$ can be reused. This strategy works, despite the dependence of $z(\theta)$ on the considered time instant, thanks to the weak degradation the convergence rate of the employed integration path experiences for time instants that are not too far apart from the one used for the definition of $z(\theta)$.

The proposed strategy is implemented as follows. Consider the $N_{\tau}$ time instants of interest to be contained in the time interval $\left[\tau_{\min }, \tau_{\max }\right]$. This time interval is then divided into octaves, with each octave $n$ covering a time subinterval $\left[\tau_{\max } / 2^{n}, \tau_{\max } / 2^{n-1}\right]$. Thus, a total number of $N_{o}=\left\lceil\ln \left(\tau_{\max } / \tau_{\min }\right) / \ln (2)\right\rceil$ octaves results, where the operator $\lceil x\rceil$ represents the least integer greater or equal than $x$. For each octave $n$, one single integration path $z_{n}(\theta)$ is used, whose parameters are computed for the reference time $\tau_{\max } / 2^{n-1}$ contained in the octave. 


\section{RESULTS AND CONCLUSIONS}

For the proposed numerical Laplace inversion formula, the total number of evaluations of the costly function $F(s)$ is $N_{o} N$, which coincides with $N_{\tau} N$ when only a single time instant of interest is contained in each octave. Figure 2 shows the relative errors committed by the numerical Laplace inversion formula (with $\varepsilon=10^{-4}$ ) in the computation of the nondimensional inlet temperature $\Theta_{\text {in }}(\tau)$ of the geothermal heat exchanger composed of 100 boreholes described in [3]. Using the same spatial resolution as in [3], the shown results required a wall-clock time of just 206 seconds using a single core of an Intel Core i7-7700K processor.

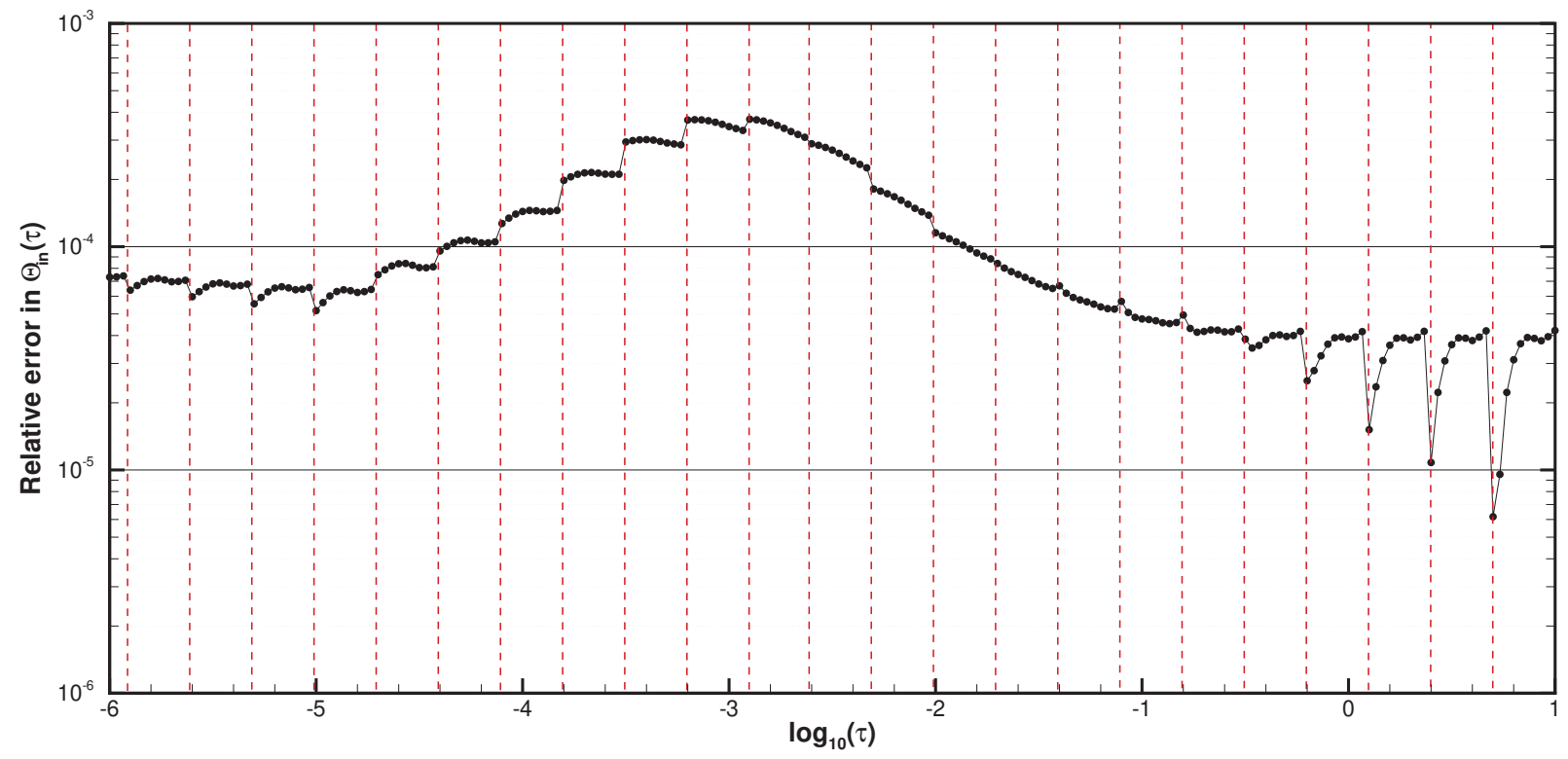

FIGURE 2. Relative error in the nondimensional inlet temperature $\Theta_{\text {in }}$ of a typical geothermal heat exchanger composed of 100 boreholes as a function of the nondimensional time $\tau$. The error level imposed onto the numerical Laplace inversion formula is $\varepsilon=10^{-4}$, and the vertical dashed lines mark the limits of the octaves used to divide the time interval $\left[\tau_{\min }, \tau_{\max }\right]=\left[10^{-6}, 10^{+1}\right]$.

\section{ACKNOWLEDGMENTS}

Work was funded by Spanish Ministerio de Economía y Competitividad through project ENE2015-68703-C2-2-R.

\section{REFERENCES}

[1] P. Eskilson, Superposition Borehole Model, Manual for Computer Code, Department of Mathematical Physics, University of Lund, Lund, Sweden (1986).

[2] P. Eskilson, "Thermal analysis of heat extraction boreholes," Ph.D. thesis, Department of Mathematical Physics, Lund Institute of Technology, Lund, Sweden 1987.

[3] M. Cimmino, International Journal of Heat and Mass Transfer 91, 1119-1127 (2015).

[4] M. Hermanns and S. Ibáñez, SIAM Journal on Applied Mathematics 79, 230-256 (2019).

[5] M. Hermanns and S. Ibáñez, Submitted to SIAM Journal on Applied Mathematics (2018).

[6] M. Hermanns, In preparation for SIAM Journal on Applied Mathematics (2019).

[7] S. Ibáñez and M. Hermanns, SIAM Journal on Applied Mathematics 78, 1658-1681 (2018).

[8] M. Hermanns, AIP Conference Proceedings 2040, 150002-1-150002-4 (2018).

[9] A. Talbot, IMA Journal of Applied Mathematics 23, 97-120 (1979).

[10] J. A. C. Weideman, SIAM Journal on Numerical Analysis 44, 2342-2362 (2006).

[11] B. Dingfelder and J. A. C. Weideman, Numerical Algorithms 68, 167-183 (2015). 